\title{
An Infrared Study of Lyman Break Galaxies in the Spitzer First Look Survey Field
}

\author{
Hyunjin Shim and Myungshin Im
}

Department of Physics \& Astronomy, FPRD, Seoul National University, Seoul, Korea email: hjshim@astro.snu.ac.kr, mim@astro.snu.ac.kr

\begin{abstract}
We investigate the properties of Lyman Break Galaxies at $z \sim 3$ in the Spitzer First Look Survey field. The effective area coverage of the study is $\sim 2.63 \mathrm{deg}^{2}$, the largest among similar studies of $R \sim 24$ mag $u$-dropouts. Photometric redshifts, ages, dust extinctions and stellar masses of the LBGs have been derived through SED fitting. More than $70 \%$ of IRAC-detected LBGs have stellar masses of order of $\sim 10^{11} \mathrm{M}_{\odot}$, comparable with $L^{*}$ galaxies. The MIR luminosity, which represents the rest-frame NIR, is found to be a good tracer of the stellar mass. As the red (optical-MIR) colors of the massive LBGs suggest, IRAC LBGs are older $(\langle t\rangle \sim 0.9$ Gyr $)$ and dustier $(\langle E(B-V)\rangle \sim 0.33)$ than typical LBGs. The age and the amount of dust indicate that the IRAC LBGs are intermediate population between blue LBG population and red DRG population. The number density of LBGs more massive than $10^{11} \mathrm{M}_{\odot}$ is $\Phi=(1.05 \pm 0.15) \times 10^{-5} \mathrm{Mpc}^{-3}$. This value exceeds the predictions of hierarchicl galaxy formation models.
\end{abstract}

Keywords. galaxies: evolution, galaxies: high-redshift, galaxies: starburst, infrared: galaxies

\section{Introduction}

The presence of massive galaxies at redshift over $z>2$ has been an interesting problem that is difficult to explain with hierarchical galaxy formation models. Although there have been many studies of UV-selected Lyman break galaxies, such studies were restricted to LBGs with mass of $\sim 10^{10} \mathrm{M}_{\odot}$ (Papovich et al. 2001; Shapley et al. 2001), much smaller than NIR selected galaxies. Recently, the addition of Spitzer data have revealed a new class of LBGs that are infrared luminous (Huang et al. 2005) and more massive than $5 \times 10^{10} \mathrm{M}_{\odot}$ (Rigopoulou et al. 2006). These massive LBGs are important tools to address how the stellar mass and stellar activity correlates and evolves along the cosmic history. However, the number of massive LBG is still small due to the small area coverage of previous studies (for e.g., $0.33 \mathrm{deg}^{2}$ in Rigopoulou et al. 2006). In this study, we enlarge the massive LBG sample and study their properties using the Spitzer First Look Survey.

\section{Properties of Massive LBGs}

The LBG "candidates" were selected with the conventional $u$-dropout method, using optical ancillary data (Fadda et al. 2004; Shim et al. 2006) of the FLS. The effective area coverage is $\sim 2.63 \mathrm{deg}^{2}$. In total, $941 u$-dropouts were selected in the central $1 \mathrm{deg}^{2}(R<$ 24.5), 173 in the outer $1.63 \operatorname{deg}^{2}(R<23.5)$. We derived the photometric redshifts, dust extinctions, and the stellar masses of the LBGs through SED fitting. The UV luminosity function of LBGs is also constructed using the photometric redshifts. Our result agrees with previous studies and provides much smaller error bars.

In the sample of 65 LBGs detected in the Spitzer IRAC bands, 50 LBGs are more massive than $10^{11} \mathrm{M}_{\odot}$. Particularly, the stellar masses of LBGs correlate well with the 

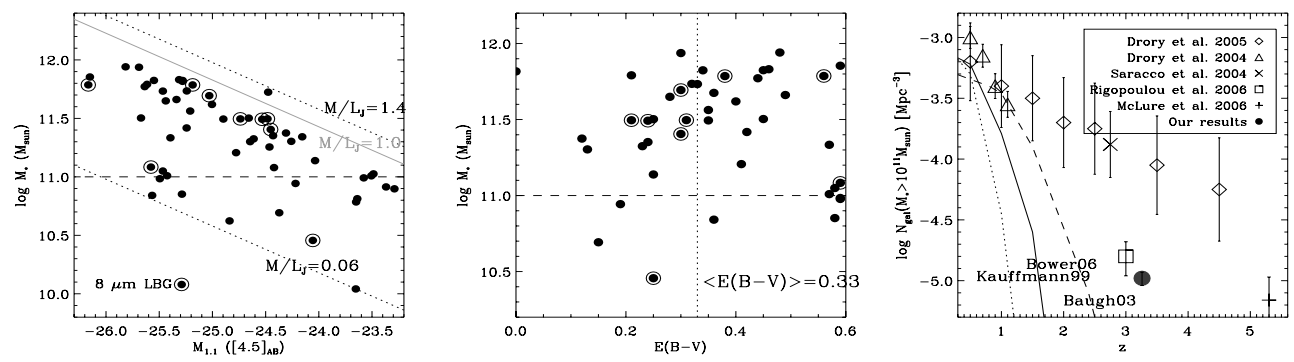

Figure 1. (a) The relation between stellar masses and $4.5 \mu \mathrm{m}$ magnitudes. (b) The relation between the amount of dust and stellar mass. (c) The number density of massive $\left(>10^{11} \mathrm{M}_{\odot}\right)$ LBGs compared with other high-redshift galaxies and the theoretical prediction for whole massive galaxies from the hierarchical galaxy formation models.

color and MIR luminosity. In Figure 1a, we show the relation between the $4.5 \mu \mathrm{m}$ (restframe $J$-band at $z \sim 3$ ) luminosities and the stellar masses. The spread in M/L ratio is $\sim 10$, and if the galaxies with large rms are excluded, the scatter in $\mathrm{M} / \mathrm{L}$ ratio is reduced to be $\sim 2$. This indicates that rest-frame NIR is a good stellar mass tracer.

These IRAC detected LBGs are found to be dustier than typical LBGs (Figure 1b). According to previous studies, typical LBGs have $E(B-V)=0.15-0.20$ (Adelberger \& Steidel, 2000). IRAC detected LBGs have a median value of $\langle E(B-V)\rangle \sim 0.33$, which is comparable with that of ILLBG extinction value of $\langle E(B-V)\rangle=0.354$ (Rigopoulou et al. 2006). These LBGs are also well-fitted by two component galaxy SED templates with $\sim 1$ Gyr old stellar population. The old age indicates that these LBGs might have formed at $z \sim 6$. The fact that IRAC detected LBGs are more massive, older, and dustier than typical LBGs suggests that these LBGs could be a bridging population between "blue" LBGs and "red" DRGs.

Finally we calculated the number density of massive $\left(>10^{11} \mathrm{M}_{\odot}\right)$ LBGs (Figure 1c). The number density $\Phi=(1.05 \pm 0.15) \times 10^{-5} \mathrm{Mpc}^{-3}$ is well above the prediction of hierarchical galaxy formation models. Comparing the number density with DRG number density, the derived fraction of LBGs among massive galaxy populations is similar with that derived from much smaller area survey (van Dokkum et al. 2006).

\section{Acknowledgements}

This work was supported in part by the BK21 program at Seoul National University.

\section{References}

Adelberger, K. L. \& Steidel, C. C. 2000, ApJ 544, 218

Fadda, D., Jannuzi, B., Ford, A., \& Storrie-Lombardi, L. J. 2004, AJ 128, 1

Huang, J.-S. et al. 2005, ApJ 634, 137

Papocivh, C., Dickinson, M., \& Ferguson, H. C. 2001, ApJ 559, 620

Rigopoulou, D. et al. 2006, ApJ 648, 81

Shapley, A. E., Steidel, C. C., Adelberger, K. L., Dickinson, M., Giavalisco, M., \& Pettini, M. 2001, ApJ 562, 95

Shim, H., Im, M., Pak, S., Choi, P., Fadda, D., Helou, G., \& Storrie-Lombardi, L. 2006, ApJS 164, 435

van Dokkum, P. G. et al. 2006, ApJL 638, 59 